\title{
A Case of Bilateral Ectopic Pregnancy Following Intra- Uterine Insemination
}

\author{
Wen Shan Simª, c, Elis Yuexian Lee ${ }^{\mathrm{b}}$, Pamela Yu Fen Tan ${ }^{\mathrm{a}}$
}

\begin{abstract}
We describe a case of a 31-year-old lady with primary subfertility who conceived only after her third intra-uterine insemination. She was diagnosed to have a right ectopic pregnancy which failed methotrexate treatment. Diagnostic laparoscopy confirmed bilateral ectopic pregnancies. A review was conducted for any consensus reached on diagnosis and optimal management of bilateral ectopic pregnancies after artificial reproductive techniques (ARTs). A search on Pubmed was carried out for publications in peer-reviewed journals with the following keywords: bilateral tubal ectopic pregnancy, heterotopic. Ten suitable publications were retrieved. Bilateral tubal ectopic pregnancy is rare, often missed on radiology and an unexpected finding at surgery. There should be a high index of suspicion for bilateral ectopic pregnancies after ART. Poor correlation with B-hCG levels further confuses diagnostic accuracy. It is prudent to ensure accurate diagnosis of bilateral ectopic pregnancies and appropriate intervention to reduce morbidity and mortality. Salpingostomy should be considered in patients with subfertility to preserve the possibility of future pregnancies.
\end{abstract}

Keywords: Artificial reproductive techniques; Bilateral tubal ectopic pregnancy; Heterotopic

\section{Introduction}

Artificial reproductive techniques (ARTs) have helped infertile couples worldwide to have children which otherwise would not have been possible. Though these new technologies provide hope and possibilities, complications of ART including ectopic pregnancies are on the rise. Ectopic pregnancies remain to be one of the main causes of maternal death and

Manuscript accepted for publication April 18, 2016

aDepartment of Obstetrics \& Gynecology, KK Women's and Children's Hospital, Singapore

${ }^{b}$ Yong Loo Lin School of Medicine, National University of Singapore, Singapore

${ }^{c}$ Corresponding Author: Wen Shan Sim, Department of Obstetrics \& Gynecology, KK Women's and Children's Hospital, 100, Bukit Timah Road, Singapore 229899, Singapore. Email: Sim.Wen.Shan@kkh.com.sg

doi: http://dx.doi.org/10.14740/jmc2484w morbidity in modern obstetrics, with a majority of these deaths associated with substandard care. Bilateral ectopic pregnancies are a rare clinical condition as a result of ART. No consensus had been reached on the diagnosis and management of bilateral ectopic pregnancies after ART because of its rarity. The following case report aimed to report the definite possibility of bilateral ectopic pregnancies from ART, highlight the difficulties in diagnosis and touch on ethical considerations in management.

\section{Case Report}

She was first seen in 2012. She was 29 years old and trying to conceive for 3 years. She had no previous pregnancies. She had a known medical history of hypothyroidism on thyroxine $100 \mu \mathrm{g}$ once a day. She reported regular periods at an interval of 28 - 30 days affected by dysmenorrhea. Her husband was a healthy 36 -year-old man who smoked $1-2$ sticks per day. She had been on a trial of ovulation induction medication for $6-7$ cycles in India with no success.

A hysterosalpingogram was performed which showed a right hydrosalpinx with loculated spill. The left fallopian tube showed intra-peritoneal spillage. Her pelvic ultrasound was normal and her anti-Mullerian hormone (AMH) level was $8.3 \mathrm{ng} / \mathrm{mL}$. Her husband's sperm analysis revealed mild hypospermia and teratozoospermia. Intra-uterine insemination (IUI) with letrozole was offered and insemination was performed on day 15 of the cycle with one mature follicle. It was unsuccessful.

The couple proceeded to have a diagnostic laparoscopy in India. Intra-operative findings were that of a clean pelvis with a patent right tube and a spasmic left tube. The couple underwent a second IUI attempt in July 2013 with ovulation induction by follicular stimulating hormone (Puregon). Three follicles more than $16 \mathrm{~mm}$ were seen on the ultrasound. She conceived after the procedure but miscarried during the first trimester.

A third IUI was performed in December 2014 after ovarian stimulation with follicular stimulating hormone (Puregon) timed with the production of two mature follicles on follicular tracking. Her hCG level 17 days after procedure was 273.3 IU/L.

She presented to the emergency unit 3 weeks after IUI with per vaginal spotting. A pelvic ultrasound showed an empty uterus with no adnexal mass. B-hCG level was 2,858 IU/L. Her second B-hCG reading $48 \mathrm{~h}$ later suboptimally increased 
Table 1. Summary of B-hCG Levels and Radiological Findings After IUI

\begin{tabular}{|c|c|c|c|c|c|}
\hline Date & 29/12/2014 & $3 / 1 / 2015$ & $5 / 1 / 2015$ & $9 / 1 / 2015$ & $15 / 1 / 2015$ \\
\hline Symptoms/events & $\begin{array}{l}\text { Day } 17 \\
\text { after IUI }\end{array}$ & $\begin{array}{l}\text { Presented with } \\
\text { vaginal spotting } \\
\text { (day } 22 \text { after IUI) }\end{array}$ & $\begin{array}{l}48 \mathrm{~h} \text { after first } \\
\mathrm{b}-\mathrm{hCG} \text { (trending) }\end{array}$ & $\begin{array}{l}\text { Day } 4 \text { after methotrexate } \\
\text { administration }\end{array}$ & $\begin{array}{l}\text { Returned to emergency } \\
\text { unit with clinical features } \\
\text { of acute abdomen. } \\
\text { Day } 10 \text { after methotrexate }\end{array}$ \\
\hline b-hCG (IU/L) & 273.3 & 2,858 & $4,123.2$ & $6,847.5$ & $1,651.8$ \\
\hline $\begin{array}{l}\text { Ultrasound } \\
\text { findings }\end{array}$ & & No IUGS seen & $\begin{array}{l}1.3 \times 0.7 \times 0.7 \mathrm{~cm} \text { ring- } \\
\text { like structure in right } \\
\text { adnexa with yolk sac. } \\
\text { No free fluid }\end{array}$ & $\begin{array}{l}1.3 \times 1.3 \times 1.1 \mathrm{~cm} \text { ring-like } \\
\text { structure in right adnexa } \\
\text { with yolk sac. No free fluid }\end{array}$ & $\begin{array}{l}\text { Free fluid in pelvis } \\
\text { Right adnexal mass } \\
1.7 \times 1.7 \mathrm{~cm}\end{array}$ \\
\hline $\begin{array}{l}\text { Management } \\
\text { options }\end{array}$ & & & $\begin{array}{l}\text { Options of conservative/ } \\
\text { surgery and methotrexate } \\
\text { offered. Patient keen } \\
\text { for methotrexate }\end{array}$ & $\begin{array}{l}\text { Option of diagnostic } \\
\text { laparoscopy offered with } \\
\text { the view of salpingostomy } \\
\text { or salpingectomy }\end{array}$ & $\begin{array}{l}\text { Option of diagnostic } \\
\text { laparoscopy offered in view } \\
\text { of hemoperitoneum from } \\
\text { ruptured ectopic pregnancy }\end{array}$ \\
\hline
\end{tabular}

to $4,123.2 \mathrm{IU} / \mathrm{L}$. A pelvic ultrasound was repeated which revealed a $1.3 \times 0.7 \times 0.7 \mathrm{~cm}$ ring-like structure containing a central cystic space with a tiny yolk sac suggestive of a right ectopic pregnancy. Options of conservative management, surgery or IM methotrexate were conveyed to the patient. Patient chose the latter and $90 \mathrm{mg}$ of IM methotrexate was administered.

Four days after methotrexate treatment, pelvic ultrasound showed the mass to be $1.3 \times 1.3 \times 1.1 \mathrm{~cm}$. No free fluid was seen. B-hCG was 6,847.5 IU/L. Patient declined any intervention at this point.

Six days later, she returned to the emergency unit with clinical features of an acute abdomen. Pelvic ultrasound showed free fluid in the pelvis and a $1.7 \times 1.7 \mathrm{~cm}$ mass in the right adnexa. She proceeded to have a diagnostic laparoscopy performed in view of the hemoperitoneum suggesting a ruptured ectopic pregnancy. A summary of her clinical presentation, B-hCG trend and radiological findings is provided in Table 1.

Diagnostic laparoscopy revealed $50 \mathrm{~mL}$ of hemoperitoneum with a large $5 \mathrm{~cm}$ right ectopic pregnancy. Right salpingectomy was performed. The left fallopian tube appeared abnormally dilated of $3 \mathrm{~cm}$ in diameter with a dusky hue and a phimosed end, visually suspected to be a left tubal ectopic pregnancy (Fig. 1). Both ovaries were normal. The husband was consulted in view of the unexpected surgical findings.
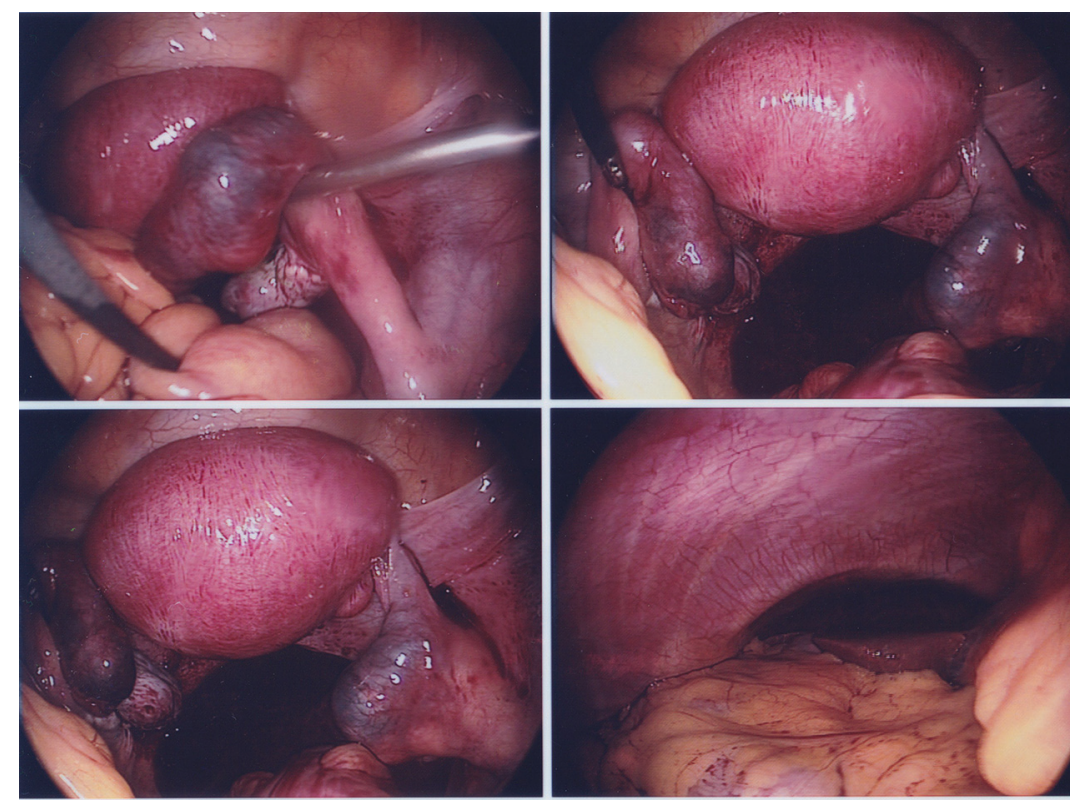

Figure 1. (Clockwise from top right) Right ectopic pregnancy; left tube with phimosed end likely ectopic pregnancy; hemoperitoneum from ruptured right ectopic pregnancy; view of the normal liver. 
Table 2. Summary of Case Reports in Terms of Their Methods of Diagnosis as Well as Subsequent Management

\begin{tabular}{lll}
\hline Title & Investigation/diagnosis & Management \\
\hline A rare case of bilateral & Initial high titres of B-hCG with a & Laparotomy with findings of \\
ectopic pregnancy & negative diagnostic evacuation of & bilateral ampullary pregnancies. \\
and differential & uterus done for suspicion of GTD. & $\begin{array}{l}\text { Bilateral salpingostomies } \\
\text { done and methotrexate }\end{array}$ \\
diagnosis of gestational & Repeated ultrasound found & administered post-operatively. \\
trophoblastic disease [1] & unilateral ectopic pregnancy. &
\end{tabular}

Heterochronic bilateral ectopic pregnancy after ovulation induction [2]

Bilateral ectopic pregnancy following ICSI [3]

Bilateral tubal ectopic pregnancy following intra-uterine insemination (IUI): a case report [4]

A case of bilateral tubal pregnancy after IVF-ET [5] (article in Bulgarian)
Ultrasound failed to show IUGS despite normal and reassuring B-hCG trend.
Patient presented with symptoms of per vaginal bleeding and right abdominal pain. TV ultrasound showed right adnexal mass suggestive of $4 \mathrm{~cm}$ right ampullary ectopic pregnancy.

\section{Laparoscopy showed $4 \mathrm{~cm}$} unruptured right ampullary ectopic pregnancy and was histologically confirmed. Left fallopian tube was inspected and deemed normal. B-hCG rose after post-operative decrease. TV ultrasound 21 days later showed left adnexa mass 2.2 $\mathrm{cm}$. IM methotrexate administrated. Three days later patient presented with hemoperitoneum. Laparoscopy confirmed a left ectopic pregnancy and a left salpingectomy was performed.

Laparoscopic salpingostomy for one side and salpingectomy for the other side.
Patient presented with pain and per vaginal Exploratory laparotomy, bleeding after IUI. Pelvic ultrasound diagnosed bilateral ectopic pregnancies with free fluid in pouch of Douglas.

left salpingectomy and right salpingostomy.
Post ET on day 36 patient presented with abdominal pain in right hypogastrium.

\author{
Bilateral ampullary \\ pregnancy after clomifen- \\ citrate and intrauterine \\ insemination - a unique \\ case report [6]
}

After clomiphene and IUI, patient presented with right abdominal pain with B-hCG 9,000 IU/L. Pelvic ultrasound showed a right adnexal mass $22 \mathrm{~mm}$.
Urgent laparotomy performed with salpingectomy due to hemoperitoneum from tubal rupture on one side. Decision undertaken for left salpingectomy after visual diagnosis of left unruptured tubal pregnancy. Histology confirmed bilateral ectopic pregnancies.

Laparoscopy for right tubal ectopic pregnancy with right salpingectomy performed. Left tube was inspected and deemed normal. Patient presented with left abdominal pain after surgery. Pelvic ultrasound revealed a left adnexal mass $17 \mathrm{~mm}$. B-hCG was 14,000 IU/L. Repeat laparoscopy done showed left ectopic pregnancy with left salpingectomy done. Histology confirmed bilateral ectopic pregnancies.

\section{Conclusion}

Laparotomy with findings of bilateral ampullary pregnancies. Bilateral salpingostomies done and methotrexate administered post-operatively.

Because of the difficulty in identification of bilateral ectopic pregnancy by ultrasonography, the clinician should be aware that the treatment of one ectopic pregnancy does not preclude the occurrence of a second ectopic pregnancy in the same patient and should pay attention to the intraoperation inspection of both side fallopian tubes in any ectopic pregnancy case.

Bilateral tubal ectopic pregnancy is a rare clinical condition. Following surgical management of this condition, it is still possible to conceive with future ICSI-ET attempts.

Cases presenting with infertility and ectopic pregnancy should be followed very closely with B-hCG and/or transvaginal sonography (TVS) to exclude double ectopic.

The frequency of some rare forms and locations of ectopic as well as heterotopic pregnancies increase after ART.

Clomifen-citrate induced cycle might result in bilateral heterotopic pregnancy not only after insemination, but also after intercourse. This condition must always be considered in emergency situations. 
Table 2. Summary of Case Reports in Terms of Their Methods of Diagnosis as Well as Subsequent Management - (Continued)

\begin{tabular}{|c|c|c|c|}
\hline Title & Investigation/diagnosis & Management & Conclusion \\
\hline $\begin{array}{l}\text { Bilateral simultaneous } \\
\text { isthmic ectopic } \\
\text { pregnancy after } \\
\text { clomiphene induction [7] }\end{array}$ & $\begin{array}{l}\text { After clomiphene and IUI, patient } \\
\text { presented with light vaginal bleeding } \\
\text { and mild abdominal pain. }\end{array}$ & & $\begin{array}{l}\text { In cases of ectopic pregnancy } \\
\text { with ovulation induced by } \\
\text { clomiphene citrate, doctors } \\
\text { must be aware of the } \\
\text { possibility of bilaterality. }\end{array}$ \\
\hline $\begin{array}{l}\text { A case report of } \\
\text { bilateral tubal ectopic } \\
\text { pregnancy following day } \\
5 \text { embryo transfer [8] }\end{array}$ & $\begin{array}{l}\text { Patient presented with vaginal bleeding } \\
\text { and abdominal pain after ICSI. Ultrasound } \\
\text { showed } 23 \mathrm{~mm} \text { left adnexal mass } \\
\text { suggestive of ectopic pregnancy. }\end{array}$ & $\begin{array}{l}\text { Laparoscopy showed } 3 \mathrm{~cm} \\
\text { unruptured left ampullary ectopic } \\
\text { pregnancy. Salpingostomy } \\
\text { performed. Inspection of right tube } \\
\text { showed bulging area in ampullary } \\
\text { region. Salpingostomy was done. } \\
\text { Histology confirmed placental } \\
\text { villi in right and left tubes. }\end{array}$ & $\begin{array}{l}\text { When a diagnosis of } \\
\text { ectopic pregnancy is } \\
\text { made, both adnexa should } \\
\text { be examined in order to } \\
\text { prevent maternal morbidity } \\
\text { and mortality. Reducing } \\
\text { the number of transferred } \\
\text { embryos can lessen bilateral } \\
\text { tubal pregnancies. }\end{array}$ \\
\hline $\begin{array}{l}\text { Combined bilateral } \\
\text { ectopic and intrauterine } \\
\text { pregnancy following } \\
\text { ovulation induction } \\
\text { with the low-dose } \\
\text { step-up protocol in a } \\
\text { patient with polycystic } \\
\text { ovary syndrome [10] }\end{array}$ & $\begin{array}{l}\text { Patient initially presented with a } \\
\text { spontaneous miscarriage, and transvaginal } \\
\text { ultrasonography revealed an empty } \\
\text { uterus. However, } 4 \text { days later, the patient } \\
\text { presented with an acute abdomen. } \\
\text { Laparoscopy revealed bilateral tubal } \\
\text { pregnancy with hemoperitoneum. } \\
\text { The right tubal pregnancy was } \\
\text { intact and the left one was ruptured } \\
\text { with extensive tubal damage. }\end{array}$ & $\begin{array}{l}\text { After a spontaneous miscarriage } \\
\text { she was treated by laparoscopic } \\
\text { left salpingectomy and right } \\
\text { linear salpingotomy. }\end{array}$ & $\begin{array}{l}\text { A woman with polycystic } \\
\text { ovary syndrome (PCOS) } \\
\text { developed bilateral tubal and } \\
\text { an intrauterine pregnancy } \\
\text { following ovulation induction } \\
\text { with urinary FSH using the } \\
\text { low-dose step-up protocol. }\end{array}$ \\
\hline
\end{tabular}

Given the background of a previous diagnostic laparoscopy in Indian showing a spasmic left tube, the options of conservative management till findings were conveyed to the patient, and left salpingostomy or salpingectomy were offered. It was discussed with the husband that since the left tube may be unhealthy, leaving it behind may risk further ectopic pregnancies and compromise future plans for in vitro fertilization. It was then decided that a left salpingectomy was to be performed. The tubes were removed and labeled separately. Histopathology returned as bilateral tubes with products of conception in keeping with bilateral ectopic pregnancy.

\section{Discussion}

With the increasing use of ARTs, the incidence of ectopic and heterotopic pregnancies has been on the rise. Bilateral ectopic pregnancy is a rare clinical condition with an estimated prevalence of $1 / 200,000$ spontaneous tubal pregnancies [1]. The prevalence is believed to be even higher in pregnancies by ART [2]. Several case reports had been published with regard to bilateral ectopic pregnancies, mostly post-ART [1-10].

Ectopic pregnancies remain to be one of the main causes of maternal death and morbidity in modern obstetrics. It is prudent to ensure early diagnosis of bilateral ectopic pregnancies with the view of early intervention to reduce morbidity and mortality for these women [9], as well as strike a balance between definitive management and future plans for conception as women who use ART are mostly those that have difficult conceiving.

Despite the number of case reports that have been published, no consensus had been reached on the diagnosis and management of bilateral ectopic pregnancies after ART. The importance of diagnosing bilateral ectopic pregnancies prior to surgical intervention could enhance pre-operative counselling in terms of suitability of tubal conservation or the need for bilateral salpingectomies as this could change the future reproductive performance of the patient. 
A search of Pubmed was carried out, not restricted by language, for publications in peer-reviewed journals with the following keywords: bilateral tubal ectopic pregnancy, heterotopic, assisted reproductive techniques. Ten suitable publications, reporting a total of 11 cases, were retrieved [1-10] ( Table 2).

We attempt to summarize the case reports in terms of their methods of diagnosis as well as subsequent management in Table 2.

There are a few learning points gathered from the 10 similar cases that we have summarized in Table 2.

1) Bilateral ectopic pregnancies are often missed on radiological investigations and are often an unexpected finding on laparoscopy/laparotomy $[4,8,10]$.

2) Extremely high B-hCG levels have poor correlation with the final diagnosis of bilateral ectopic pregnancies [1], although differentials of bilateral ectopic pregnancies should be considered on top of other conditions such as a molar pregnancy [3].

3) There should be a high index of suspicion for bilateral ectopic pregnancies after pregnancies conceived after ART for both clinicians and radiologists [5], including pregnancies arising from clomiphene citrate $[6,7]$.

4) For pregnancies after ART with a diagnosed unilateral ectopic pregnancy going for surgery, a close inspection at the contralateral tube [8] during laparoscopy/laparotomy is crucial as ultrasonography for early ectopic pregnancies may be difficult [4].

5) Salpingostomy should be considered for unruptured bilateral ectopic pregnancies [9] in patients with history of subfertility to preserve the possibility of future pregnancies. Patients should be counselled on the pros and cons of salpingostomy which include need for longer follow-up for persistent trophoblastic disease as well as recurrent ectopic pregnancies.

From our case study, we also extrapolated that failed methotrexate treatment in an ectopic pregnancy which arises after ART may signify the possibility of bilateral ectopic pregnancy. This information should be provided to the radiologist or sonographer so that a closer look at the contralateral tube can be performed $[5,8,9]$.

\section{Conclusion}

Ectopic pregnancy is a gynecological emergency. Due to the use of assisted reproductive techniques, the incidence of bilateral ectopic pregnancies is on the rise. It is often only diagnosed during laparoscopy [4], as it may be missed on transvaginal sonography. Beta-hCG trends can be misleading [1]. No consensus has been reached with regard to diagnosis and management of bilateral tubal ectopic pregnancies. Salpingostomy should be considered for unruptured bilateral ectopic pregnancies [9] in patients with history of subfertility to preserve the possibility of future pregnancies. Successful conception is still possible in the future with IVF treatment, even in patients who underwent bilateral salpingectomies [1]. Future studies can look into how to enhance diagnosis of bilateral ectopic pregnancies pre-operatively, so as to optimize management of this condition. Ethical considerations include obtaining valid consent for management of an unexpected finding of a bilateral ectopic pregnancy during surgery which will impact on a woman's future reproductive performance. We believe that this case would add value to the growing literature on bilateral ectopic pregnancies following ARTs.

\section{Conflicts of Interest}

The authors have no conflicts of interest relevant to this article.

\section{References}

1. Polat M, Boynukalin FK, Yarali I, Yarali H. Bilateral ectopic pregnancy following ICSI. BMJ Case Rep. 2014;2014.

2. Kovachev E, Kozovski I, Ivanov S, Kornovski Y, Mircheva N, Koleva P, Tsonev A, et al. [A case of bilateral tubal pregnancy after IVF - ET]. Akush Ginekol (Sofiia). 2013;52(4):33-37.

3. Arab M, Neda Kazemi S, Vahedpoorfard Z, Ashoori A. A rare case of bilateral ectopic pregnancy and differential diagnosis of gestational trophoblastic disease. J Reprod Infertil. 2015;16(1):49-52.

4. Zhu B, Xu GF, Liu YF, Qu F, Yao WM, Zhu YM, Gao HJ, et al. Heterochronic bilateral ectopic pregnancy after ovulation induction. J Zhejiang Univ Sci B. 2014;15(8):750755.

5. Jamilian M. Bilateral tubal ectopic pregnancy following intra uterine insemination (IUI): A case report. Iran J Reprod Med. 2014;12(2):155-158.

6. Terzic MM, Bila JS, Pilic IZ, Kocijancic DM. Bilateral ampulary pregnancy after clomifen citrate and intrauterine insemination - a unique case report. Gynecol Endocrinol. 2013;29(6):619-621.

7. Pehlivanov BK, Amaliev GI, Malinova ML, Amaliev IG. Bilateral simultaneous isthmic ectopic pregnancy after clomiphene induction. Folia Med (Plovdiv). 2012;54(4):78-79.

8. Ghaffari F, Eftekhari Yazdi P, Kiani K. A case report of bilateral tubal ectopic pregnancy following day 5 embryo transfer. Arch Med Sci. 2011;7(6):1087-1088.

9. Altinkaya SO, Ozat M, Pektas MK, Gungor T, Mollamahmutoglu L. Simultaneous bilateral tubal pregnancy after in vitro fertilization and embryo transfer. Taiwan J Obstet Gynecol. 2008;47(3):338-340.

10. Yarali H, Bukulmez O, Gurgan T. Combined bilateral ectopic and intrauterine pregnancy following ovulation induction with the low-dose step-up protocol in a patient with polycystic ovary syndrome. Arch Gynecol Obstet. 2000;264(1):37-38. 